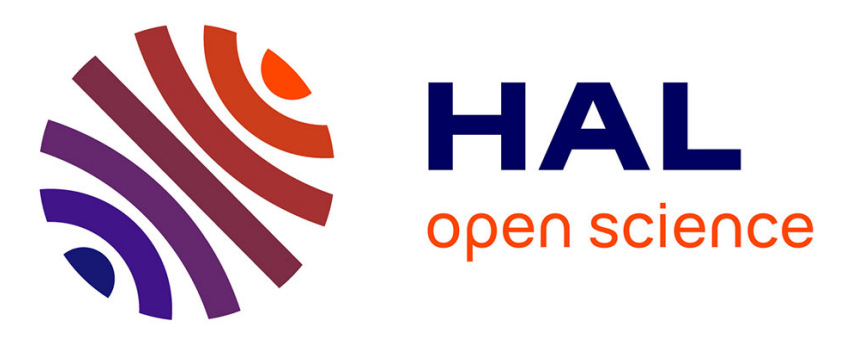

\title{
The Double Drum Peel (DDP) test: a new concept to evaluate the delamination fracture toughness of cylindrical laminates
}

Federica Daghia, Christophe Cluzel, Louis Hébrard, François Churlaud, Benoît Courtemanche

\section{To cite this version:}

Federica Daghia, Christophe Cluzel, Louis Hébrard, François Churlaud, Benoît Courtemanche. The Double Drum Peel (DDP) test: a new concept to evaluate the delamination fracture toughness of cylindrical laminates. Composites Part A: Applied Science and Manufacturing, 2018, 113, pp.83-94. hal-01770031

\section{HAL Id: hal-01770031 \\ https://hal.science/hal-01770031}

Submitted on 18 Apr 2018

HAL is a multi-disciplinary open access archive for the deposit and dissemination of scientific research documents, whether they are published or not. The documents may come from teaching and research institutions in France or abroad, or from public or private research centers.
L'archive ouverte pluridisciplinaire HAL, est destinée au dépôt et à la diffusion de documents scientifiques de niveau recherche, publiés ou non, émanant des établissements d'enseignement et de recherche français ou étrangers, des laboratoires publics ou privés. 


\title{
The Double Drum Peel (DDP) test: a new concept to evaluate the delamination fracture toughness of cylindrical laminates
}

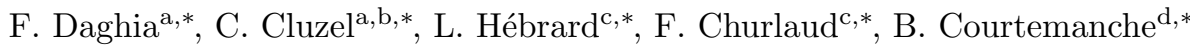 \\ ${ }^{a}$ LMT, ENS de Cachan, CNRS, Univ. Paris-Saclay \\ 61 av. du Président Wilson, F-94235 Cachan CEDEX, France \\ ${ }^{b} I U T$ d'Evry Val d'Essonne, Département SGM, Bâtiment Maupertuis \\ 3 rue du Père Jarlan, 91025 Evry CEDEX, France \\ ${ }^{c} D G M$, ENS de Cachan, Univ. Paris-Saclay \\ 61 av. du Président Wilson, F-94235 Cachan CEDEX, France \\ ${ }^{d}$ CETIM, Technocampus Composites \\ Chemin du Chaffault, 44340 Bouguenais, France
}

\begin{abstract}
Delamination testing of monolithic composites is typically carried out using configurations in which the crack is a symmetry plane for both overall geometry and stacking sequence, in order to ensure a controlled crack tip mode ratio. These normalized configurations do not enable testing of curved specimens, like those manufactured by filament winding. To overcome this limit, we propose a new concept for the delamination testing of cylindrical laminates, the Double Drum Peel test. A global energy analysis of the DDP test, including all sources of energy release and dissipation, is carried out to provide a simple formula for the fracture toughness. The local mode mixity is evaluated based on analytical results on the classical peel test. Finally, some tests using carbon-peek rings manufactured by laser assisted tape placement are carried out on a prototype machine to illustrate the potential of this new concept.
\end{abstract}

Keywords: B. Delamination; D. Mechanical testing; E. Filament Winding; E. Tape placement

\section{Introduction}

Delamination, that is the propagation of cracks in the weak, matrix-rich area between plies, is a typical and extremely dangerous mode of failure of laminated composites. For this reason, the experimental characterization and the modeling of delamination has been a constant preoccupation for the academic and industrial worlds in the last decades. Currently, the typical approach to characterize the delamination resistance of an 'interface' between plies is to evaluate the fracture toughness $G_{c}$ for the three different modes of crack propagation ( $G_{I c}$ for the opening mode, $G_{I I c}$ and $G_{I I I c}$ for the shearing modes) and for different mixed-mode combinations. Indeed, the energy dissipated during delamination propagation strongly depends on the loading conditions, since different local dissipation mechanisms can be activated (see for example [1]). A mixed-mode criterion

\footnotetext{
*Corresponding author. Tel. +33 (0)1 474028 31, Fax +33 (0)1 47402785.

Email addresses: federica.daghia@ens-paris-saclay.fr (F. Daghia),

christophe.cluzel@ens-paris-saclay.fr (C. Cluzel), louis.hebrard@ens-cachan.fr (L. Hébrard),

francois. churlaud@ens-cachan.fr (F. Churlaud), benoit.courtemanche@cetim.fr (B. Courtemanche)
} 
can then be defined and it is easily integrated in one of the typical modeling approaches used for crack propagation, such as those based on Linear Elastic Fracture Mechanics or on Cohesive Zone Models.

Obtaining experimental values for pure-mode fracture toughness, however, imposes significant restrictions on the specimen geometry and testing configuration. The overall geometry and the stacking sequence of the specimen are generally required to be symmetric with respect to the crack plane, and the symmetric or antisymmetric portions of the loading conditions determine the mode I and mode II contributions to the overall dissipated energy. Further requirements on the stacking sequence of each portion of the specimen after delamination can also be included (see $[2,3])$ in order to prevent the release of residual stresses, as well as the dissipation of energy within the plies: all of the energy is thus released by the delamination crack, and a global measurement of the strain energy release rate during propagation enables one to identify the critical strain energy release rate of the interface.

The standard delamination tests currently available $[4,5,6,7,8]$ are all based on this principles. Their strict symmetry requirements, however, prevent the testing of a range of potentially interesting configurations:

- delamination of an $[\alpha / \beta]$ interface between plies with different orientation: even though specific layups can be conceived, whose overall stiffness enables one to satisfy all of the requirements at the scale of the homogeneous plate (see for example $[2,3]$ ), the local symmetry at the scale of the ply is broken because of the different orientations of the adjacent plies;

- delamination which does not occur along the mid-plane of the specimen, such as skin-core debonding in sandwich panels;

- delamination of curved specimens, such as those manufactured by filament winding.

While a number of literature studies exist which consider $[\alpha / \beta]$ interfaces (see for example $[2,9]$ ), thus neglecting the local symmetry requirement, very few studies have considered more macroscopic symmetry losses. Among the notable exceptions is [10] and the references therein: these works discuss Double Cantilever Beam (DCB), End Notched Flexure (ENF) and Mixed Mode Bending (MMB) tests on specimens obtained by longitudinally cutting filament wound composite tubes, which therefore present a curvature in the width direction. In all of these cases, the experimental results can be treated to yield a fracture toughness value, however it is not clear how this value should be used with respect to the classical pure-mode fracture toughnesses defined in the normalized characterization.

Some other interesting ideas for non symmetric testing configurations can be found in the literature concerning the characterization of adhesives. Many similarities exist between delamination and the failure of adhesive bonds (for an interesting comparison between the two communities, see the common publication [11]). In adhesives, the substrates, or adherends, are often made of differ- 
ent materials, have different thicknesses or non-flat geometries, thus quasi-systematically breaking the symmetry of the problem. In this case, the typical approach to characterize the bond quality is to evaluate a fracture force or moment (see for example the ASTM standard for the Climbing Drum Peel (CDP) test, [12]): these values can only be used to compare the relative strength of different adhesives, since they depend on the geometry of the testing setup. An alternative approach, closer in principle to the delamination characterization in composites as described above, is to use the same test and an energy analysis to extract a global fracture toughness from test data. In this case, a significant difference with the approach described for composites is that the different propagation modes are generally not distinguished (that is, only a single $G_{c}$ value is measured), the underlying hypothesis being that only a single mechanism governs the fracture behavior. Eventually, a distinction can be made between cohesive failure, where the crack propagates within the adhesive layer, and adhesive failure, where the crack propagates along the adherend/adhesive interface, and two different values of $G_{c}$ can be defined. A third possible type of failure, which is generally not sought after in peel tests, corresponds to the crack deviating into the adherent (substrate failure).

Recently, some testing concepts typical of adhesives have been applied to delamination testing of monolithic composites [3, 13]. In [3], the CDP test is revisited for monolithic laminates and its results for different $[\alpha / \beta]$ interfaces are compared to those obtained by DCB. In particular, the global results in terms of fracture toughness $G_{c}$ obtained in the two tests were observed to be correlated to the local results in terms of fracture surfaces (and thus of local dissipation mechanisms): fracture surfaces having similar microscopic features yielded similar $G_{c}$ values, irrespective of the macroscopic test configuration. Non pure-mode loading conditions appear therefore to be pertinent for the characterization of fracture toughness, provided the possibility to make a link, at least qualitative, with the local dissipation mechanisms. The quantitative link between the global toughness and the local dissipation mechanisms, however, is still a long-term future development.

In this paper, we propose a new concept for the experimental characterization of the delamination behavior of cylindrical specimens. The Double Drum Peel (DDP) test concept, described in Section 2, was initially developed by Cetim for the comparative characterization of the bonding quality in Laser Assisted Tape Placement rings as a function of the processing parameters. In order to extend its use to characterization of the delamination behavior of LATP and filament wound specimens, a global energy analysis is carried out in Section 3 to provide a simple formula for the computation of the critical fracture toughness from test data. Furthermore, the link between the testing configuration and the local mode mixity is discussed in Section 4, based on analytical results on the classical variable angle peel test. Finally, in Section 5 some experimental tests using carbon-peek rings manufactured by LATP are carried out on a prototype machine recently patented by Cetim $[14,15]$ to illustrate the potential of this new testing concept. A short summary and some perspectives conclude the paper. 


\section{The Double Drum Peel test concept}

The Double Drum Peel (DDP) test concept was originally developed at Cetim. A schematic illustration of the test, as well as a photography of the prototype machine developed and patented by Cetim, are shown in Figure 1.

The test specimen is a laminated composite ring, manufactured by circumferential winding of a pre-impregnated tape around a mandrel of radius $R_{1}$; the last portion of the tape is left unattached from the rest of the specimen, thus constituting the pre-delamination required for typical peel and delamination propagation tests. This top layer, which will be detached from the rest of the specimen during the test, is named 'peel arm' hereafter.

Two cylindrical supports, or drums, of radii $R_{1}$ and $R_{2}$, each one of which can rotate around its center $O_{1}$ and $O_{2}$, constitute the core of the testing machine. In order to simplify matters, $R_{1}=R_{2}=R$ is often considered. In the prototype developed by Cetim, $R=100 \mathrm{~mm}$. The test specimen is clamped to the drum 1 (on the left in the pictures), while the end of the peel arm is clamped to the drum 2 .

The drum 2 is then set in motion. A constant angular velocity $\dot{\alpha}_{2}>0$ (in the clockwise direction in the pictures) is imposed and the couple $C_{2}>0$ applied by the motor on the drum to maintain this velocity is measured: this rotation pulls on the peel arm, thus eventually causing the delamination to propagate. For a simple analogy, think of a portion of scotch tape being peeled from its dispenser.

This simple configuration would not enable one to control the angle at which peeling occurs (the 'peel angle', defined as $\beta$ in Figure 1a). Indeed, as in the scotch tape analogy, the peel angle would tend to $\beta=90^{\circ}$ if there is no significant friction, in order to maximize the effectiveness of the peel force, as it will be clarified in the global energy model in Section 3. A large peel angle is not necessarily optimal, as the significant bending occurring in the vicinity of the tip of the delamination crack may cause other forms of damage and, thus, pollute the results of the test, or even prematurely break the peel arm. For this reason, a resisting couple $C_{1}<0$ is applied to the drum 1 using a feedback control loop, which enables one to choose and maintain a constant and suitable peel angle $\beta$ throughout the test. The angle $\beta$, required for the control loop, is measured continuously during the test thanks to a measuring arm posed on the tape, which can be seen in Figure 1b.

The original aim of this test concept, as developed by Cetim, was the comparative characterization of the quality of the bond between a pre-impregnated carbon/thermoplastic tape and its substrate in rings manufactured by Laser Assisted Tape Placement. In particular, the measure of the couples and of the peel angle enables one to calculate the peel force ( $F$ in Fig. 1a) as:

$$
F=-\frac{C_{1}}{\left(R_{1}+h_{r}+\frac{h_{p}}{2}\right) \cos \beta}=\frac{C_{2}}{\left(R_{2}+\frac{h_{p}}{2}\right)}
$$

where $h_{p}$ and $h_{r}$ are the thickness of the peel arm and of the rest of the specimen, and the local 
bending in the vicinity of the drum 2 has been neglected. By setting a constant peel angle $\beta$ and comparing the peel forces associated to specimens manufactured using different process parameters, a comparative evaluation of the bond quality as a function of the manufacturing process could be obtained. In the following, the same test concept is revisited in order to characterize the delamination behavior of cylindrical specimens.

\section{A global model of the DDP test}

A global characterization of the delamination behavior of composites requires to define a procedure to extract the overall fracture toughness, or critical energy release rate $G_{c}$, from the measured test data. In this Section, we establish an energy balance in order to define a general expression for the critical strain energy release rate. We then particularize this expression to the case of the DDP test, specifically analyzing the different sources of released and dissipated energy which may be present in the system. Finally, we introduce some simplifying hypotheses, leading to a more compact expression of the critical strain energy release rate which will be used in the analysis of the tests presented in Section 5 .

\subsection{Energy balance}

The concept of an energy criterion controlling crack propagation was originally introduced in the 1920s by Griffith in his seminal paper [16], and reformulated in the context of the thermodynamic of irreversible processes in the 1970s. The formulation given in the following is not the only possible one. Its choice is oriented by the motivation of this work, which aims at characterizing the dissipation associated to the propagation of a crack along the interface between two plies and, thus, considers all of the other dissipation sources as parasite phenomena, or losses. These losses need to be controlled, or at least quantified, in order to extract an intrinsic value for the fracture toughness of the interface to be characterized.

Starting from the first and second principle of thermodynamics applied to the whole system under quasi-static and isothermal conditions, and supposing that loading and crack surface are the only two parameters which describe its evolution, the dissipation $D$ can be written as

$$
D=-\frac{\partial E_{p}}{\partial A} \dot{A} \geq 0
$$

where $E_{p}$ is the potential energy of the system and $A$ is the crack surface area. This enables us to define the expression of the strain energy release rate $G$ as

$$
G=-\frac{\partial E_{p}}{\partial A}=-\frac{\partial\left(E_{d}-W_{e x t}\right)}{\partial A}
$$

where $E_{d}$ is the elastic strain energy stored in the system and $W_{\text {ext }}$ is the work of the external forces. 
The dissipation related to the creation of new cracked surfaces is assumed to be defined by a material parameter $G_{c}$, the critical strain energy release rate, so that

$$
D_{\text {crack }}=G_{c} \dot{A}
$$

which is the material quantity that we seek to determine. It should be noted that $G_{c}$ is a macroscopic quantity associated to the 'interface' between two plies, and it is constituted by a variety of microscopic dissipation phenomena. Defining which phenomena should be included within $G_{c}$ is a matter of choice and it depends on the final aim of the experimental characterization. Here, we choose to define $G_{c}$ based on the dissipation occurring in the vicinity of the 'interphase', that is the thin layer between the two plies (such as local matrix plasticity, fiber/matrix debonding, ...) at the crack tip and in the crack process zone. In particular, the crack process zone is supposed to be small and close to the interphase. This enables us to define an intrinsic value of the interface fracture toughness $G_{c}$, which can then be used for predictive computations based on Linear Elastic Fracture Mechanics or Cohesive Zone Models.

In general, other sources of energy dissipation, or losses, can be present in the system, which we qualify here as $D_{l}$. This term is voluntarily unspecified here: it contains all of the parasite dissipation phenomena (friction, damage, anelasticity...) which can occur within the system and which may, if neglected, lead to an overestimation of the value of the critical strain energy release rate. The specific sources of dissipation which may be present in the DDP test are discussed in Section 3.2. In accordance with the hypotheses introduced earlier, the term $D_{l}$ also assumed proportional to the newly created crack surface, as

$$
D_{l}=G_{l} \dot{A}
$$

This leads to the following reformulation of Eq. (2):

$$
D=G \dot{A}=\left(G_{c}+G_{l}\right) \dot{A} \geq 0
$$

The crack propagation model can then be expressed as follows

$$
f=G-\left(G_{c}+G_{l}\right) \leq 0, \quad \dot{A} \geq 0, \quad f \dot{A}=0
$$

which leads, during crack propagation at $f=0$, to the following expression for $G_{c}$ :

$$
G_{c}=G-G_{l}=-\frac{\partial\left(E_{d}-W_{e x t}\right)}{\partial A}-G_{l}
$$

\subsection{Energy balance for the DDP test}

The different energy terms discussed in the previous Section are evaluated here for the DDP test, considering steady-state propagation. 


\subsubsection{Hypotheses and kinematic description}

The peel arm is modeled as a beam of width $b$ and thickness $h_{p}$, which can be divided into three zones:

1. the undelaminated zone, which is still attached to the rest of the composite ring around the drum 1;

2. the zone which is wound around the drum 2 ;

3. the zone between the two drums, comprising a central straight portion and two lateral bent portions.

The test is driven by imposing the angular velocity $\dot{\alpha}_{2}$ of the drum 2 . An increment $\mathrm{d} \alpha_{2}$ of its rotation corresponds to an increment $\mathrm{d}_{2}$ in the length of zone 2 of the peel arm, as:

$$
\mathrm{d} \alpha_{2}=\chi_{2} \mathrm{~d} l_{2}=\frac{\mathrm{d} l_{2}}{R_{2}+\frac{h_{p}}{2}}
$$

where $\chi_{2}$ is the curvature of the peel arm in zone 2 and $R_{2}$ is the radius of the drum 2 .

When the delamination propagates, the drum 1 is set in motion. An increment $\mathrm{d} \alpha_{1}$ of its rotation corresponds to an decrement $\mathrm{d} l_{1}$ in the length of zone 1 of the peel arm, as:

$$
\mathrm{d} \alpha_{1}=\chi_{1} \mathrm{~d} l_{1}=\frac{\mathrm{d} l_{1}}{R_{1}+h_{r}+\frac{h_{p}}{2}}
$$

where $\chi_{1}$ is the curvature of the peel arm in zone $1, R_{1}$ is the radius of the drum 1 and $h_{r}$ is the thickness of the composite ring without the peel arm.

It should be underlined that, in general, neither $\left(\mathrm{d} l_{1}, \chi_{1}\right)$ or $\left(\mathrm{d} l_{2}, \chi_{2}\right)$ are stress-free configurations for the peel arm: indeed, zone 1 may be subjected to residual stresses, while elastic stresses are present in zone 2 . The reference stress-free configuration is defined here as $\left(\mathrm{d} l_{0}, \chi_{0}\right)$.

\subsubsection{Cracked area}

Assuming a self-similar propagation of the crack front, the increment of the cracked area is expressed as

$$
\mathrm{d} A=b \mathrm{~d} l
$$

where $b$ is the width of the peel arm and $\mathrm{d} l$ is the increment in length of the crack.

If the term $h_{p} \chi_{0}$ is small, we have $\mathrm{d} l \approx \mathrm{d} l_{0}$, where the delaminated length $\mathrm{d} l$ is measured along the intrados of the peel arm and the length $\mathrm{d} l_{0}$ is measured along its axis.

\subsubsection{Work of external forces}

The evolution of the work of external forces $\mathrm{d} W_{e x t}$ is given by the two applied couples as

$$
\mathrm{d} W_{e x t}=C_{1} \mathrm{~d} \alpha_{1}+C_{2} \mathrm{~d} \alpha_{2}
$$

It should be noted that the term $C_{1} \mathrm{~d} \alpha_{1}$ of Eq. (12) gives a negative contribution: indeed, $\mathrm{d} \alpha_{1}$ and $\mathrm{d} \alpha_{2}$ are both positive, whereas, as explained in Section 2, we have $C_{1}<0$. 


\subsubsection{Elastic energy}

The elastic strain energy $E_{d}$ is stored in the peel arm, as well as in the rest of the specimen and of the experimental setup. Only the elastic energy stored in the peel arm, however, evolves during crack propagation: for this reason, only the peel arm needs to be modeled to compute $\mathrm{d} E_{d}$.

The material is supposed to be in its elastic domain in zones 1 and 2, while dissipation in the form of damage can occur in zone 3, particularly due to significant bending in the vicinity of the drum 1. Zones 1 and 2, whose lengths change during steady-state delamination, contribute to determine the term $\mathrm{d} E_{d}$ of the energy balance which is discussed here. Zone 3 , whose length and shape are constant during steady-state delamination, may contribute to the dissipation term $G_{l} \mathrm{~d} A$ discussed in Section 3.2.5.

Zone 1. Residual strain/stress. The undelaminated portion of the peel arm may be subjected to residual stresses, which are released during delamination and thus should be estimated in order to carry out a proper energy balance. These residual stresses are generally unknown and they derive from a variety of sources: the tension applied to the composite mesh during layup, the difference between the stress-free temperature and the service temperature, the chemical shrinkage during polymerization of thermoset-based composites, the crystallization shrinkage during cooling of thermoplastic-based composites.

In accordance with the beam model defined for the peel arm in this work, the residual stresses are partitioned here into a membrane and a bending term, denoted as $N_{r}$ and $M_{r}$, respectively. When the delamination propagates, these stresses are released in a portion of peel arm of stress-free length $\mathrm{d} l_{0}$, giving rise to the following terms:

$$
\begin{aligned}
\mathrm{d} E_{d, r t} & =-\frac{1}{2} \frac{N_{r}^{2}}{(E S)_{e q, 1}} \mathrm{~d} l_{0}=-\frac{1}{2}(E S)_{e q, 1} \varepsilon_{r}^{2} \mathrm{~d} l_{0} \\
\mathrm{~d} E_{d, r b} & =-\frac{1}{2} \frac{M_{r}^{2}}{(E I)_{e q, 1}} \mathrm{~d} l_{0}=-\frac{1}{2}(E I)_{e q, 1} \chi_{r}^{2} \mathrm{~d} l_{0}
\end{aligned}
$$

where $(E S)_{e q, 1}$ and $(E I)_{e q, 1}$ are the membrane and bending stiffnesses of the peel arm in zone 1, $\varepsilon_{r}$ and $\chi_{r}$ are the residual membrane strain and the residual curvature, respectively.

The residual terms are responsible for the difference between the configurations $\left(\mathrm{d} l_{1}, \chi_{1}\right)$ and $\left(\mathrm{d} l_{0}, \chi_{0}\right)$ : in particular, we have

$$
\varepsilon_{r}=\frac{\mathrm{d} l_{1}-\mathrm{d} l_{0}}{\mathrm{~d} l_{0}}, \quad \chi_{r}=\chi_{1}-\chi_{0}
$$

Zone 2. Elastic strain/stress. During the DDP test, zone 2 of the peel arm is subjected to the peel force $F$, as well as to bending moments which enable its winding around the drum 2 .

The peel force $F$ during delamination depends on the peel angle $\beta$ and on the fracture toughness $G_{c}$ of the interface to be tested. While it is not measured directly during the DDP test, its value can be retrieved by equilibrium considerations (see Section 3.3.1). The membrane contribution to the evolution of the elastic strain energy when the delamination propagates of a length $\mathrm{d} l_{0}$ thus 
reads:

$$
\mathrm{d} E_{d, t}=\frac{1}{2} \frac{F^{2}}{(E S)_{e q, 2}} \mathrm{~d} l_{0}=\frac{1}{2}(E S)_{e q, 2} \varepsilon_{e}^{2} \mathrm{~d} l_{0}
$$

where $(E S)_{e q, 2}$ is the membrane stiffness of the peel arm in zone 2 and $\varepsilon_{e}$ is the elastic membrane strain. Note that $(E S)_{e q, 2}$ may be different from $(E S)_{e q, 1}$ due to the damage which may develop in zone 3 .

The bending contribution, on the other hand, is related to the difference between the curvature $\chi_{2}$ around the drum 2 and the stress-free curvature $\chi_{0}$. The elastic curvature $\chi_{e}$ thus reads:

$$
\chi_{e}=\chi_{2}-\chi_{0}=\frac{M_{e}}{(E I)_{e q, 2}}
$$

where $(E I)_{e q, 2}$ is the bending stiffness of the peel arm in zone 2, which may be different from $(E I)_{e q, 1}$ due to damage developing in zone 3. During propagation, this gives rise to the following term:

$$
\mathrm{d} E_{d, b}=\frac{1}{2} \frac{M_{e}^{2}}{(E I)_{e q, 2}} \mathrm{~d} l_{0}=\frac{1}{2}(E I)_{e q, 2} \chi_{e}^{2} \mathrm{~d} l_{0}
$$

The elastic terms are responsible for the difference between the configurations $\left(\mathrm{d} l_{2}, \chi_{2}\right)$ and $\left(\mathrm{d} l_{0}, \chi_{0}\right)$ : in particular, we have

$$
\varepsilon_{e}=\frac{\mathrm{d} l_{2}-\mathrm{d} l_{0}}{\mathrm{~d} l_{0}}, \quad \chi_{e}=\chi_{2}-\chi_{0}
$$

\subsubsection{Energy losses from other sources of dissipation}

As it was pointed out in Section 3.1, the creation of new cracked surfaces may not be the only dissipation mechanism occurring during the test. Other mechanisms, defined here as energy losses, can include plasticity, damage, friction... In the following discussion, we will distinguish in particular:

1. the dissipation mechanisms within the specimen (peel arm and remaining portion of the ring);

2. the dissipation mechanisms in the rest of the experimental setup, which will be supposed here to be negligible.

The dissipation mechanisms within the specimen should be considered with special care. In adhesives, it is quite easy to distinguish between the adhesive layer and the substrates, or adherends, and thus to attribute a given dissipation mechanism to the one or the other. If a mechanism is associated to the adhesive layer, or to the bond between the adhesive and the adherend, the energy it dissipates is part of the global cohesive or adhesive fracture toughness of the characterized interface. If a mechanism is associated to one of the adherends, on the other hand, it constitutes a separate energy loss, which should not be included in the fracture toughness and, thus, needs to be separately evaluated. A typical example of the second kind of phenomena is the plastic deformation in a metallic peel arm due to bending of the peel arm in the vicinity of the crack tip: this term is 
often the main source of energy dissipation in the peel tests involving metallic adherends, failing to account for it separately would lead to an extreme overestimation of the fracture toughness of the characterized system.

In laminated composites, the distinction between the mechanisms associated to the delaminated interface and to the rest of the specimen is less obvious, and thus slightly more arbitrary, as it was discussed previously in the paper. When observed at a fine scale, cracks initiated in the matrixrich layer between plies can propagate to involve plastic dissipation within the matrix, fiber/matrix debonding, fibers pull-out... For the purposes of this global analysis, all of these mechanisms will be included in the fracture toughness of the interface if they occur in the vicinity of the crack tip and along the crack process zone. Similar phenomena occurring farther away from the interphase, on the other hand, are considered to be energy losses within the peel arm or in the rest of the specimen.

Looking at the overall specimen response during a delamination test, it is generally not possible to distinguish between the energy dissipated in the interface and the other energy losses. Different strategies can therefore be adopted:

- the first possibility is to reproduce the same experimental conditions, but in the absence of delamination propagation, in order to experimentally quantify the energy losses and subtract them from the overall dissipated energy. This is the approach retained, for example, in the CDP test setup, where the winding of the pre-delaminated portion of the specimen serves as a baseline for the computation of the energy dissipated during delamination (see [3]);

- the second possibility is to model each of the energy losses: the dissipation associated to each mechanism can then be identified under experimental conditions which can be different from those of the delamination test, and the energy losses in the delamination testing conditions can be obtained by simulation. This is the approach retained here.

The dissipation mechanisms which may need to be modeled include fiber/matrix debonding, transverse matrix cracking, local fiber kinking and fiber breaking. The first two mechanisms concern the matrix or the fiber/matrix interface, and they generally occur in plies subjected to in-plane transverse or shear loading. The third and fourth mechanisms involve the fibers, and they can occur due to compressive and tensile loading in the fibers' direction, respectively. The peel arm is essentially loaded in tension along the fibers' direction, but it includes a high bending region in the vicinity of the drum 1. Local failures involving kinking and isolated fiber breaking could be expected in this region, which should be carefully inspected after the test.

\subsection{Critical strain energy release rate expression for the DDP test}

The energy balance developed in Section 3.1, together with the specification of each energy term defined in Section 3.2, are used here to define the expression of the critical strain energy release rate as measured by the DDP test. 
Replacing the terms derived in Section 3.2 into Eq. (8) yields:

$$
\begin{aligned}
G_{c} & =\frac{C_{1}\left(1+\varepsilon_{r}\right)}{b\left(R_{1}+h_{r}+\frac{h_{p}}{2}\right)}+\frac{C_{2}\left(1+\varepsilon_{e}\right)}{b\left(R_{2}+\frac{h_{p}}{2}\right)} \\
& +\frac{1}{2 b}\left[(E S)_{e q, 1} \varepsilon_{r}^{2}+(E I)_{e q, 1} \chi_{r}^{2}-(E S)_{e q, 2} \varepsilon_{e}^{2}-(E I)_{e q, 2} \chi_{e}^{2}\right]-G_{l}
\end{aligned}
$$

While it may not be apparent from Eq. (20), this expression of the critical strain energy release rate bears many similarities to the one associated to the classical variable angle peel test [17]. Furthermore, it can be highly simplified by neglecting some of the contributions, thus leading to a much simpler expression, whose validity should nevertheless be verified by estimating the order of magnitude of the neglected terms. This two points are discussed in the next Sections.

\subsubsection{Analogy with the classical variable angle peel test formula}

A schematic view of the classical variable angle peel test is given in Fig. 2. Assuming a homogeneous, elastic peel arm of Young's modulus E, and neglecting the residual stresses and energy loss terms, the critical strain energy release rate is derived by Kendall [17] as

$$
G_{c}=\frac{F}{b}(1-\cos \beta)+\left(\frac{F}{b}\right)^{2} \frac{1}{2 E h_{p}}
$$

In particular, the first term is related to the work of external forces only (supposing the peel arm inextensible), while the second term is related to the elastic energy stored in the peel arm itself.

In order to compare this expression to the one obtained for the DDP test, Eq. (20) is reformulated in terms of the peel force using Eq. (1). This yields:

$$
\begin{aligned}
G_{c} & =-\frac{F}{b}\left(1+\frac{N_{r}}{E h_{p} b}\right) \cos \beta+\frac{F}{b}\left(1+\frac{F}{E h_{p} b}\right) \\
& +\frac{1}{2 b}\left[\frac{N_{r}^{2}}{E h_{p} b}+\frac{E b h_{p}^{3}}{12} \chi_{r}^{2}-\frac{F^{2}}{E h_{p} b}-\frac{E b h_{p}^{3}}{12} \chi_{e}^{2}\right]-G_{l}
\end{aligned}
$$

where the elastic and residual strain are formulated in terms of peel force and residual membrane force and the membrane and bending stiffnesses are specialized for a homogeneous beam of Young's modulus E. Simplification and rearrangement yields

$$
\begin{aligned}
G_{c} & =\frac{F}{b}(1-\cos \beta)+\left(\frac{F}{b}\right)^{2} \frac{1}{2 E h_{P}} \\
& +\left(\frac{N_{r}}{b}\right)^{2} \frac{1}{2 E h_{P}}-\frac{F N_{r}}{E h_{p} b^{2}} \cos \beta \\
& +\frac{1}{2} \frac{E h_{p}^{3}}{12}\left[\chi_{r}^{2}-\chi_{e}^{2}\right]-G_{l}
\end{aligned}
$$

As it can be noticed, the first line of Eq. (23) is identical to Eq. (21) describing the classical peel test. The second line of Eq. (23) is related to the residual membrane strains in the peel arm: while they are usually not accounted for in the peel test modeling (and, thus, they do not appear in Eq. (21)), they are analogous to those later derived by Thouless and Jensen [18] for the classical peel test including residual membrane strains. Finally, the last line of Eq. (23) contains the bending 
elastic and residual terms, which do not apply to the classical peel test, as well as the other energy losses, which are usually neglected in the modeling of the classical peel test.

\subsubsection{A simplified formula for the computation of fracture toughness in the DDP test}

As it was pointed out earlier, the complete formula in Eq. (20) or Eq. (23) can be greatly simplified by introducing some further simplifying hypotheses.

The bending contributions are proportional to the term $E h_{p}^{3} \chi^{2}$. The peel arm should be flexible enough in order to be bent around the drums without inducing damage or failure: for a given Young's modulus $E$, this can be achieved by choosing a thin peel arm and large radii of curvature. In this case, the bending terms become small. Furthermore, the residual and elastic terms give contributions of opposite signs, and thus nearly cancel each other for similar curvatures $\chi_{e}$ and $\chi_{r}$, that is for $R_{1}+h_{r}+\frac{h_{p}}{2} \approx R_{2}+\frac{h_{p}}{2}$.

The residual membrane term $N_{r}$ depends on the manufacturing process: for filament winding and Laser Assisted Tape Placement (LATP), it is essentially related to the tension in the yarn during fibers' placement, thus it is relatively small and it will be neglected in the following.

The relative importance of the membrane elastic energy in the peel arm versus the external work contribution depends on the peel angle $\beta$. The ratio between the two energy terms reads:

$$
\text { Ratio }=\frac{\frac{F}{b} \frac{F}{2 E b h_{p}}}{\frac{F}{b}(1-\cos \beta)}=\frac{\frac{\varepsilon_{e}}{2}}{1-\cos \beta}
$$

It should be underlined that the peel force $F$, or its dimensionless counterpart the elastic strain $\varepsilon_{e}$, is not an independent variable: assuming that $G_{c}$ does not depend on the testing configuration (an assumption which will be challenged later in the paper), the peel force at delamination increases as the peel angle decreases.

In Figure 3, the ratio between the elastic energy and the external work is plotted as a function of the peel angle $\beta$ for different values of dimensionless peel force. As it can be seen, the elastic contribution is always dominant for very small peel angles (for $\beta=0$, in particular, the external work contribution vanishes), but it becomes small for increasing peel angles, especially if the peel force is small. Considering that the peel force at delamination increases as the peel angle decreases, the tendency observed in each curve is exacerbated when considering the energy ratio during delamination.

Choosing a very small peel angle for the DDP test may not be feasible, since the peel force required to delaminate may exceed the tensile strength of the peel arm: the peel arm may break in tension before delamination could occur. For the carbon/PEEK composite tested in the following, the testing range starts around $\beta>20^{\circ}-30^{\circ}$. For the same reason, the elastic strain should remain below the elastic limit for the material under consideration (for carbon fiber composites, $\varepsilon_{e, \max } \approx 0.01$ ). Within this range, the elastic contribution is at most around $10 \%$ percent (see Figure 3). 
The energy losses due to damage depend on the amount of local bending near the crack tip, which increases for large peel angles. Choosing a very large peel angle for the DDP test may not be possible either, since the local applied moment during delamination may exceed the bending strength of the peel arm, breaking it before delamination could occur. For the carbon/PEEK composite tested in the following, the testing range ends around $\beta<50^{\circ}-60^{\circ}$. Within this range, the local bending contribution to energy dissipation should be evaluated by visual inspection and, eventually, characterization of the bending behavior of the peel arm.

Under the described hypotheses, it is possible to define a simplified formula for $G_{c}$ which considers only the external work contribution to the strain energy release rate:

$$
G_{c}=\frac{C_{1}}{b\left(R_{1}+h_{r}+\frac{h_{p}}{2}\right)}+\frac{C_{2}}{b\left(R_{2}+\frac{h_{p}}{2}\right)}
$$

If the two cylinders have equal radii $R_{1}=R_{2}=R$ and the specimen and peel arm thicknesses $h_{r}$ and $h_{p}$ are small compared to $R$ we can further simplify to

$$
G_{c}=\frac{C_{1}+C_{2}}{b R}
$$

This simplified expression was used to analyze the DDP tests described in Section 5. Interestingly enough, in terms of the peel force $F$ we have

$$
G_{c}=\frac{F}{b}(1-\cos \beta)
$$

which is identical to the typical simplified expression for the classical peel test.

\section{Local loading conditions in the peel test: a discussion on the mode mixity}

The global energy analysis carried out in Section 3 enabled us to provide a full, as well as a simplified expression for the critical strain energy release rate associated to crack propagation within the DDP test. The purely energetic approach, however, provides no information concerning the loading conditions in the vicinity of the crack, and in particular on the mode mixity, which is the classical way to characterize the crack tip loading. As it was discussed in the Introduction, the energy dissipated during crack propagation strongly depends on the loading conditions of the interface. For this reason, the mode mixity associated to peel tests, an in particular its variation as a function of the peel angle, is discussed in this Section.

As it was pointed out in Section 3.3.1, a strong analogy can be drawn between the DDP and the classical variable angle peel test. For this reason, the discussion carried out in this Section is essentially based on analytical results developed in the literature for the classical peel test. In particular, we will consider a two-dimensional peel problem, thus exploring the partition between modes I and II in the central portion of the specimen and neglecting the effect of the lateral free surfaces on the mode mixity. 


\subsection{Mode mixity: definition and limitations}

The partition of a cracking problem into mode I and mode II components can be defined:

- in terms of displacement, based on the direction of the relative displacements of the points on either side of the crack with respect to the crack plane;

- in terms of stress, based on the direction of the singular stresses in front of the crack tip with respect to the crack plane.

In both cases, the direction orthogonal to the crack plane defines the mode I (opening) component, while the direction parallel to the crack plane defines the mode II (shearing) component. These definitions were originally developed for homogeneous and isotropic material. Their extension to multi-material systems and different classes of material symmetries is not obvious, since the displacement-based and the stress-based definitions are not analogous [19].

In the following discussion, a homogeneous and isotropic material is considered in order to make use of results available in the literature [18, 22]. Even so, analytical results defining the mode mixity as a function of loading only exist for a limited number of relatively simple configurations.

\subsection{Mode mixity in the classical variable angle peel test}

Let us consider a semi-infinite homogeneous and isotropic solid with Young's modulus $E$ and Poisson's coefficient $\nu$, with a planar crack positioned at a distance $h_{p}$ from the surface. The elastic behavior is defined by the term $\bar{E}$, where $\bar{E}=E$ in plane stress and $\bar{E}=\frac{E}{1-\nu^{2}}$ in plane strain conditions. The strain energy release rates and the mode ratio associated to remote axial, bending and shear loading conditions (that is $N, M$ and $V$ as depicted in Figure 4) are given as [19, 20, 21]:

$$
G=\left(\frac{N}{b}\right)^{2} \frac{1}{2 \bar{E} h_{p}}+\left(\frac{M}{b}\right)^{2} \frac{6}{\bar{E} h_{p}^{3}}+\left(\frac{V}{b}\right)^{2} \frac{f_{v}^{2}}{\bar{E} h_{p}}
$$

and

$$
\frac{G_{I I}}{G_{I}}=\left(\frac{K_{I I}}{K_{I}}\right)^{2}=\left(\frac{-N h_{p} \sin \omega-M \sqrt{12} \cos \omega+V h_{p} \sqrt{2} f_{v} \sin \psi_{v}}{-N h_{p} \cos \omega+M \sqrt{12} \sin \omega+V h_{p} \sqrt{2} f_{v} \cos \psi_{v}}\right)^{2}
$$

where $\omega=52.07^{\circ}[20], f_{v}^{2}=3.764$ and $\psi_{v}=0.7^{\circ}$ [21] in a homogeneous, isotropic material. Here, $K_{I}$ and $K_{I I}$ are the stress intensity factors in mode II and mode I, respectively, which are typically used to define the mode mixity. Contrary to the strain energy release rates, which is always positive, they are signed quantities. In particular, solutions having $K_{I}<0$ should be discarded since this means that the crack is closed, whereas the sign of $K_{I I}$ defines the direction in which the crack tends to kink during propagation.

Expressing $N, V$ and $M$ in terms of the peel force $F$ and of the peel angle $\beta$ enables one to compute the mode mixity of the classical peel test using Eq. (29). In particular, $N$ and $V$ can be easily determined from equilibrium considerations:

$$
N=F \cos \beta, \quad V=F \sin \beta
$$


while $M$ is obtained by equating Eqs. (28) and (21) (where $\bar{E}$ replaces $E$ in general plane elasticity).

The mode mixity of the classical peel test is analyzed in the following.

\subsubsection{Mode mixity for constant peel force}

The role of the peel force $F$ and of the peel angle $\beta$ in determining the mode mixity of the peel test is investigated here. As it was stated before in the paper, the peel force is not an independent variable: its value at delamination increases as the peel angle decreases for a constant critical strain energy release rate $G_{c}$.

To simplify the comparison between different materials and geometries, the dimensionless elastic tensile strain $\varepsilon_{e}=\frac{F}{E b h_{p}}$ in the peel arm is taken as a parameter. The mode ratio $G_{I I} / G_{I}$ is plotted in Figure 5 as a function of the peel angle $\beta$ for different values of $\varepsilon_{e}$.

For small peel forces, both the shear and the axial contributions rapidly become negligible for increasing peel angles. The results is a mode mixity completely controlled by bending. Although it may not appear intuitive, a pure bending moment does not yield pure mode I conditions due to the lack of geometric symmetry, but it encompasses a significant contribution of mode II: indeed, for the limit case of $\varepsilon_{e}=0$ we have $G_{I I} \approx 0.6 G_{I}$.

For large peel forces, the axial and shear terms become more significant. The axial term is dominant at small peel angles. It gives both mode I and mode II contributions due to the lack of symmetry. In particular, the mode I contributions of the axial and bending terms are of opposite sign, meaning that the (negative) axial contribution tends to close the crack. Eventually, the overall mode I contribution becomes zero, thus making the mode ratio tend to infinity. The shear term, on the other hand, is dominant at large peel angles. Its contribution is essentially in mode I, thus it decreases the mode ratio with respect to pure bending solution.

As it was pointed out previously in the paper, the range of peel angles and peel forces which can be encountered in practice is limited by the tensile and bending strength of the peel arm. For the carbon/PEEK composite tested in the following $\left(20^{\circ}<\beta<60^{\circ}, \varepsilon_{e, \text { max }} \approx 0.01\right)$ the mode mixity varies between 0.4 and 0.9 .

\subsubsection{Mode mixity for constant critical strain energy release rate}

In order to plot the evolution of the mode ratio at delamination, it is necessary to fix a value of the critical strain energy release rate $G_{c}$, as well as a criterion for crack propagation in mixed mode conditions. Here, we define the dimensionless quantity $g_{c}=\frac{G_{c}}{E h_{p}}$ and we consider a mode independent crack propagation, for the sake of simplicity. With the typical orders of magnitude we have:

- for thermoset matrix composites:

$$
g_{c}=\frac{10^{2} \mathrm{~J} / \mathrm{m}^{2}}{10^{11} \mathrm{~Pa} \cdot 10^{-4} \mathrm{~m}}=10^{-5}
$$


- for thermoplastic matrix composites:

$$
g_{c}=\frac{10^{3} \mathrm{~J} / \mathrm{m}^{2}}{10^{11} \mathrm{~Pa} \cdot 10^{-4} \mathrm{~m}}=10^{-4}
$$

The mode ratio as a function of peel angle for different values of $g_{c}$ is plotted in Fig. 6 . For the testing range and $g_{c}$ values considered in the following, the mode mixity is nearly constant with the peel angle at around $G_{I I} \approx 0.6 G_{I}$. This is an interesting result, as it enables one to choose the peel angle based on other considerations, such as the desire to limit the degradation in the peel arm associated to bending for large peel angles.

\subsection{Limitations of the present analysis with respect to the DDP test}

The analytical results presented here are very simple to exploit, and therefore they can be used to rapidly compute the mode mixity for the specific material, geometrical and loading conditions encountered in a given DDP test. In order to fulfill this objective, however, we had to accept some simplifications:

- all of the results discussed here concern a crack in a homogeneous, isotropic material: orthotropy, or the presence of different materials (adherends and/or an interphase material) are not accounted for;

- the model describes mode mixity for the classical variable angle peel test configuration, and not the DDP configuration: in particular, the eventual effect of curvature of the peel arm and substrate on the mode mixity is not accounted for;

- the loading condition at the crack tip is described in terms of mode mixity in the sense of Linear Elastic Fracture Mechanics, implying a crack process zone of zero length: if the size of the crack process zone is not negligible, it should be taken into account in the description of the local loading conditions (see for example the discussion in [23]).

A more accurate analysis could be carried out numerically, using the exact geometry, material properties and loading conditions of an actual DDP test, and evaluating the local loading conditions through approaches such as the Virtual Crack Closure Techniques (VCCT) for LEFM, or Cohesive Zone Models (CZM) to account for the crack process zone. This constitutes obviously a much more cumbersome analysis, and a loss of generality with respect to the results presented here.

\section{Some experimental results}

The DDP test was carried out on several carbon-peek rings manufactured by Laser Assisted Tape Placement (LATP). Different testing configurations are considered, with peel angles going from $\beta=30^{\circ}$ to $\beta=50^{\circ}$, which highlight different phenomena occurring along the delaminated interface and within the peel arm. The simplified post-treatment formula given in Eq. (26) is used to compute the critical strain energy release rate for all of the tests. 


\subsection{Description of the specimens and of the DDP tests}

Six composite rings were manufactured at the Cetim facility in Nantes, using a pre-impregnated carbon/PEEK thermoplastic tape of nominal width $b=14 \mathrm{~mm}$ and nominal thickness $h_{p}=0.16$ $\mathrm{mm}$. Each manufactured ring had a nominal thickness of $h_{r}=0.96 \mathrm{~mm}$ and an internal radius of $R=100 \mathrm{~mm}$. The manufacturing parameters were kept constant for all of the rings.

The prototype testing machine patented by Cetim was used to carry out the DDP tests. Each DDP test requires only a relatively short delaminated length to measure stable values of the couples $C_{1}$ and $C_{2}$ necessary for the computation of the critical strain energy release rate using Eq. (26). For this reason, multiple tests could be carried out on each ring, in order to assess the repeatability and the effect of the peel angle within a single specimen, and not only across specimens. In particular, each ring was tested at increasing peel angles in a range between $\beta=30^{\circ}$ and $\beta=50^{\circ}$. For all tests, the imposed delamination velocity was of $20 \mathrm{~mm} / \mathrm{min}$.

\subsection{Results}

The direct output of each DDP test is the measurement of couples $C_{1}<0$ and $C_{2}>0$. The simplified formula Eq. (26) is used to compute the critical strain energy release rate.

Three examples of raw measurements of $C_{1}$ and $C_{2}$ for different peel angles are reported in Figure 7. In particular, the solid lines represent $C_{2}$, while the dashed lines represent $-C_{1}$, so that the distance between these two lines is directly proportional to the critical strain energy release rate in the delamination phase of the test. The sum $C_{1}+C_{2}$ is plotted directly in Figure 8 for the same three tests.

All of the test curves have an analogous structure. The first portion of the curve, with increasing couples, corresponds to the transient phase during which the peel arm is put into tension. Then comes the steady-state portion of the test, during which delamination occurs: during this phase, the two couples are expected to be nearly constant for constant interface properties. The average value of the sum $C_{1}+C_{2}$ is evaluated and used to compute the critical strain energy release rate of the interface (see Figure 8 for the definition of the portions over which the averages were computed).

Comparing the results of the three tests, one can notice that the absolute value of couples $C_{1}$ and $C_{2}$ is higher for the tests with smaller peel angle, while the value of the sum $C_{1}+C_{2}$ is rather similar between the different tests. The couples $C_{1}$ and $C_{2}$ are directly proportional to the peel force $F$, which increases as the peel angle decreases. Using Eq. (1), we can evaluate the peel force for each test: in particular, in this range of peel angles we have $60 \mathrm{~N}<F<200 \mathrm{~N}$, thus $10^{-4}<\varepsilon_{e}<10^{-3}$. The results of Section 4 enable us to evaluate the mode mixity, which is constant at $G_{I I}=0.6 G_{I}$ within this range of peel angles and elastic strains. The critical strain energy release rate is therefore expected to be constant across the tests, as is the value of $C_{1}+C_{2}$, which is directly proportional to $G_{c}$. 
While the curves for $\beta=40^{\circ}$ display very smooth and rather constant couple measurements, the curves for $\beta=30^{\circ}$ and $\beta=50^{\circ}$ have some peculiar features, which were observed in many of the repeated tests at the same peel angles. The sudden drop in load observed for the curve for $\beta=30^{\circ}$ corresponds to an unstable propagation of the delamination along a finite distance. A photograph of the delaminated surface is given in Figure 9: the lighter portions of the surface, which could be observed for the tests at every peel angle, correspond to the progressive delamination, whereas the darkest portion, which could only be found in tests where the load drops occurred, corresponds to the unstable jump. The presence of these unstable propagations reduces the portion of the curve which can be exploited for the identification of the critical strain energy release rate. For this reason, small peel angles are not ideal even before exceeding the tensile failure load of the peel arm. The curves for $\beta=50^{\circ}$ also show some irregularities, namely two sudden load increases at the beginning of the test, followed by a decrease in the values of the couples and of their difference as the test progresses. The $C_{1}+C_{2}$ curve, proportional to $G_{c}$, is also lower than the ones corresponding to the other peel angles. The reason for this behavior can be understood by inspecting the peel arm after the test. In Figure 10, one can observe significant damage: in particular, fibers failures (probably triggered by local bending) and transverse cracks have led to the separation of some meshes from the bulk of the peel arm. The reduction in the delaminated area brought about by this detachment is believed to be responsible of the decrease in the $G_{c}$ value, which was computed using the total width $b$ of the peel arm. The peel angle $\beta=50^{\circ}$ appears to induce unwanted damage in the peel arm, therefore it is not ideal either for the determination of the delamination fracture toughness.

The post-treated results in terms of $G_{c}$ for all of the tests which were carried out are plotted in Figure 11 as a function of the peel angle. In particular, each marker represents a different specimen (numbered 2222 to 2227), since multiple DDP tests were carried out on the same specimen. The values of $G_{c}$ appear rather repeatable across specimens and for different peel angles. An exception is the specimen n. 2222, which appears to be systematically tougher than the others: this may be due to differences in the material or the manufacturing process, even though the nominal conditions were the same. The $G_{c}$ values at a peel angle of $\beta=50^{\circ}$ are lower than the others, which could be related to the reduction in the delaminated section as observed in the specimen depicted in Figure 10: for this reason, these values are deemed to be less reliable. Overall, a value of $G_{c} \sim 2 \mathrm{~kJ} / \mathrm{m}^{2}$ is coherent with typical values for carbon/PEEK composites.

\section{Conclusions and perspectives}

A new concept for the delamination testing of cylindrical laminates was proposed in this paper. The Double Drum Peel (DDP) test was presented and analyzed in details, both from a global energy perspective and in terms of the local mode mixity. A simplified formula for the computation of the critical strain energy release rate from test data was obtained, and the mode mixity was 
shown to be nearly constant in the range of peel angles and for the types of materials considered. Some experimental results on carbon/PEEK cylinders shows the potential of this method for the characterization of specimens manufactured by Laser Assisted Tape Placement or filament winding.

Current work in progress involves the comparison of the DDP, CDP and DCB test results. The three tests will be carried out on specimens produced using the same material and processing conditions. They will be compared in terms of global results, that is the measured values of the fracture toughness $G_{c}$, as well as of local indicators, that is the fracture surfaces and their microscopic features, which are related to the local dissipation mechanisms during delamination.

\section{References}

[1] E. S. Greenhalgh, C. Rogers, P. Robinson, Fractographic observations on delamination growth and the subsequent migration through the laminate, Compos Sci Technol 69 (2009) 2345-2351.

[2] X. J. Gong, A. Hurez, G. Verchery, On the determination of delamination toughness by using multidirectional DCB specimens, Polym Test 29 (2010) 658-666.

[3] F. Daghia, C. Cluzel, The Climbing Drum Peel (CDP) test: an alternative to the Double Cantilever Beam (DCB) for the determination of fracture toughness of monolithic laminates, Compos: Part A 78 (2015) 70-83.

[4] ISO-15024, Fibre-reinforced plastic composites - determination of mode I interlaminar fracture toughness, GIC, for unidirectionally reinforced materials (2002).

[5] ASTM-D5528, Standard test method for mode I interlaminar fracture toughness of unidirectional fiber-reinforced polymer matrix composites.

[6] ISO-15114, Fibre-reinforced plastic composites - determination of the mode II fracture resistance for unidirectionally reinforced materials using the calibrated end-loaded split (C-ELS) test and an effective crack length approach (2014).

[7] ASTM-D7905, Standard test method for determination of the mode II interlaminar fracture toughness of unidirectional fiber-reinforced polymer matrix composites (2014).

[8] ASTM-D6671, Standard test method for mixed mode I-mode II interlaminar fracture toughness of unidirectional fiber reinforced polymer matrix composites (2013).

[9] O. Allix, D. Lévêque, L. Perret, Identification and forecast of delamination in composite laminates by an interlaminar interface model, Compos Sci Technol 58 (5) (1998) 671-678.

[10] P. Davies, L. A. Carlsson, Major Accomplishments in Composite Materials and Sandwich Structures: An Anthology of ONR Sponsored Research, Springer, 2009, Ch. Delamination of composite cylinders, pp. $67-82$. 
[11] D. R. Moore, A. Pavan, J. G. Williams (Eds.), Fracture Mechanics Testing Methods for Polymers Adhesives and Composites, Elsevier, 2001.

[12] ASTM-D1781, Standard test method for climbing drum peel for adhesives (1998).

[13] W. J. B. Grouve, L. L. Warnet, R. Akkerman, Critical assessment of the mandrel peel test for fiber reinforced thermoplastic laminates, Eng Fract Mechan 101 (2013) 96-108.

[14] B. Courtemanche, L. Fradin, P.-Y. Josselin, Méthode et dispositif d'essai de pelage de couches de matériau en bande, Patent FR3025316, filed August 28, 2014, issued December 09, 2016.

[15] B. Courtemanche, L. Fradin, P.-Y. Josselin, Peel test method and device for layers of material in tape form, Patent WO2016030641, filed August 28, 2015.

[16] A. A. Griffith, The phenomena of rupture and flow in solids, Philosophical Transactions of the Royal Society of London. Series A, Containing Papers of a Mathematical or Physical Character 221 (1921) 163-198.

[17] K. Kendall, Thin-film peeling - the elastic term, J Phys D Appl Phys 8 (1975) 1449-1452.

[18] M. D. Thouless, H. M. Jensen, Elastic fracture mechanics of the peel-test geometry, J Adhesion 38 (1992) 185-197.

[19] J. W. Hutchinson, Z. Suo, Advances in Applied Mechanics (Book 29), Academic Press, 1991, Ch. Mixed mode cracking in layered materials.

[20] Z. Suo, J. W. Hutchinson, Interface crack between two elastic layers, Int J Fracture 43 (1990) $1-18$.

[21] S. Li, J. Wang, M. D. Thouless, The effects of shear on delamination of beam-like geometries, J Mech Phys Solids 52 (2004) 193-214.

[22] M. D. Thouless, Q. D. Yang, A parametric study of the peel test, Int J Adhes Adhes 28 (2008) $176-184$.

[23] L. De Lorenzis, G. Zavarise, Debonding analysis of thin plates from curved substrates, Eng Fract Mech 77 (2010) 3310-3328. 


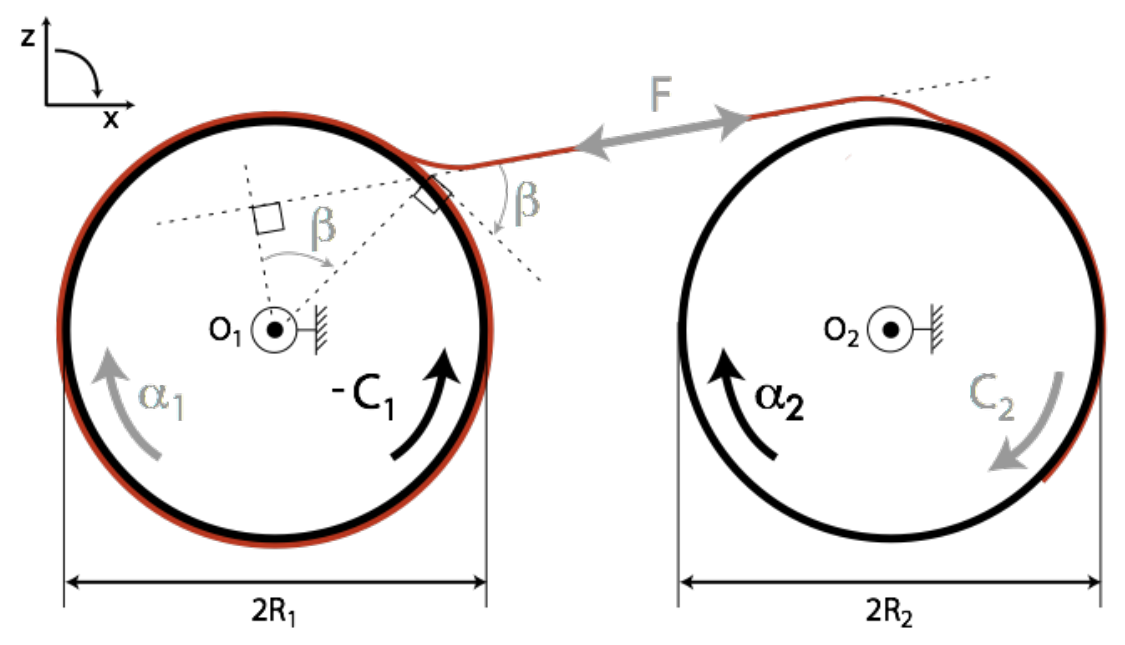

(a) The test concept: the specimen is depicted in red, the imposed quantities $\alpha_{2}$ and $C_{1}<0$ are in black, the measured quantities $C_{2}, \alpha_{1}, \beta$ and the computed peel force $F$ are in grey

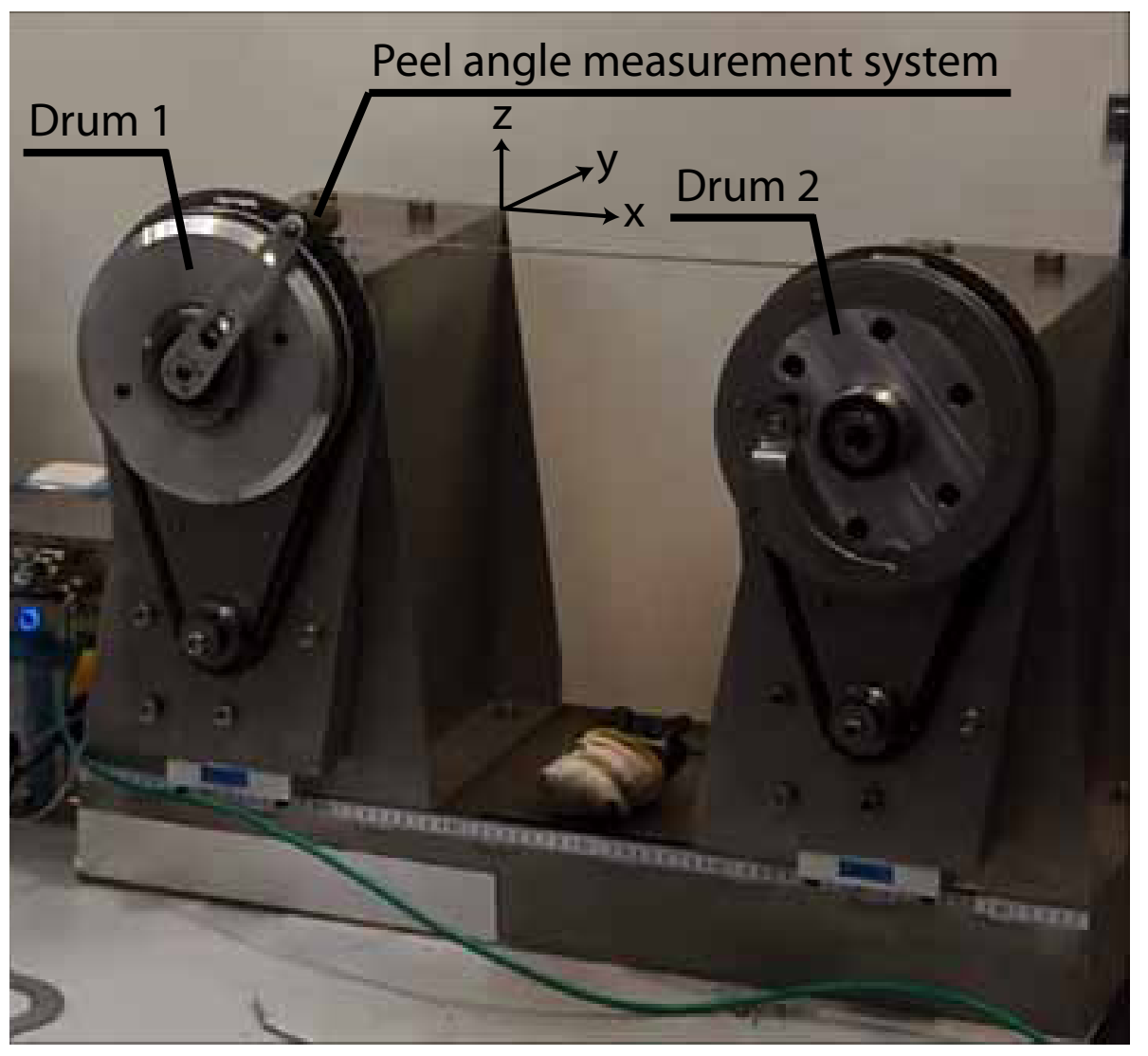

(b) The prototype developed and patented by Cetim

Figure 1: The DDP test 


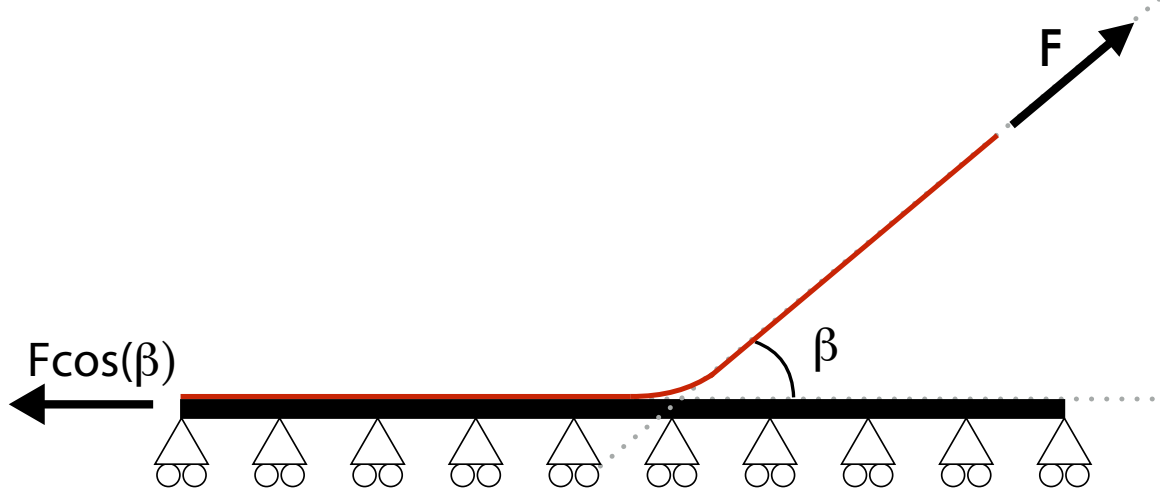

Figure 2: Schematic illustration of the classical variable angle peel test concept.

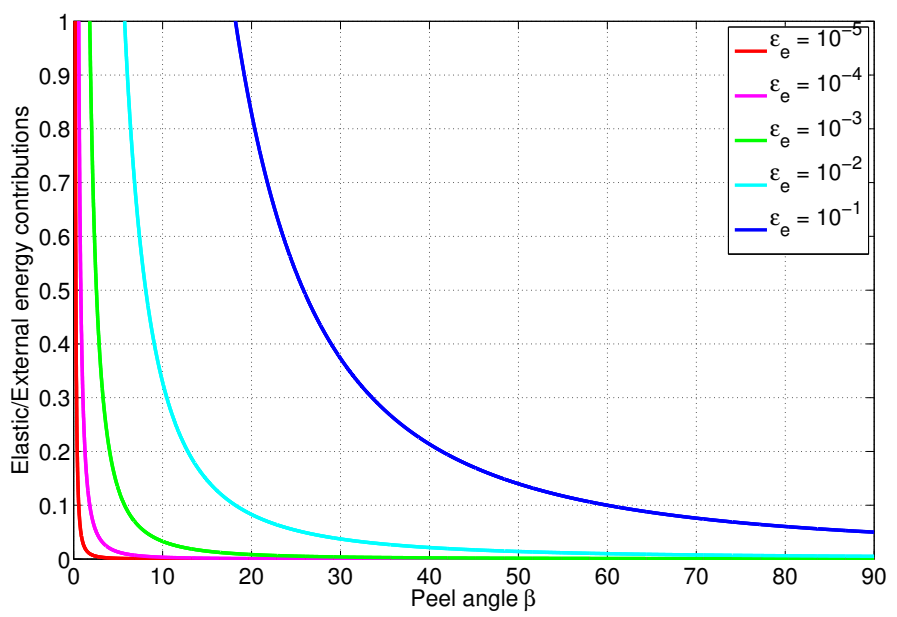

Figure 3: Ratio between the elastic and the external work contributions to the strain energy release rate as a function of the peel angle for different values of dimensionless peel force 


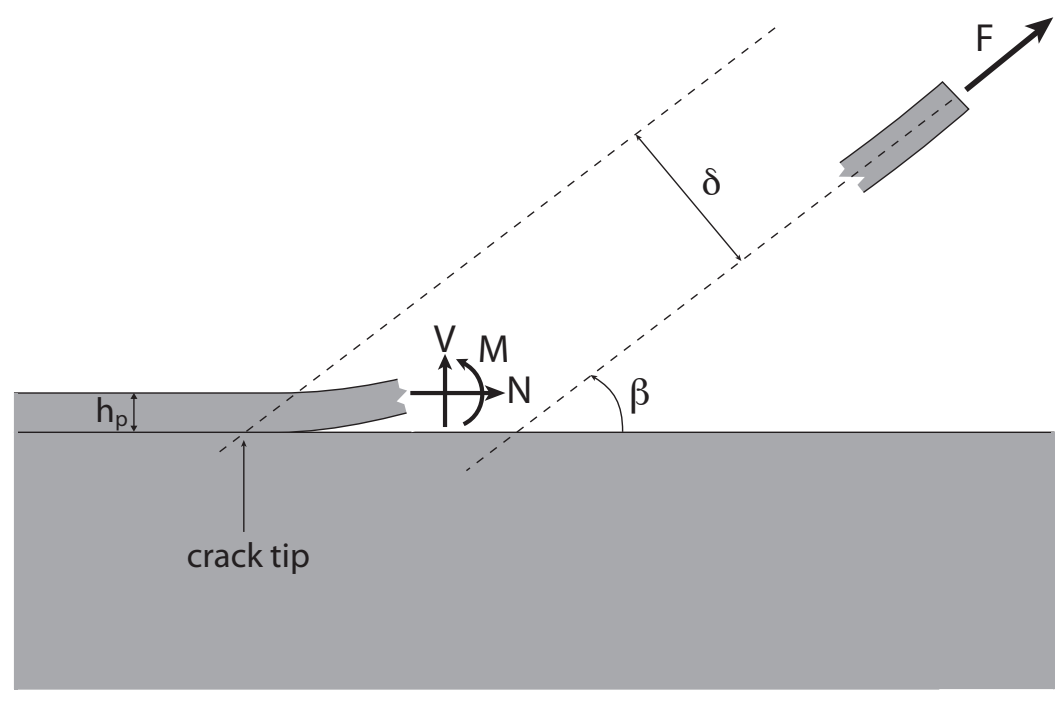

Figure 4: Axial, shear and bending loading conditions for the classical variable angle peel test

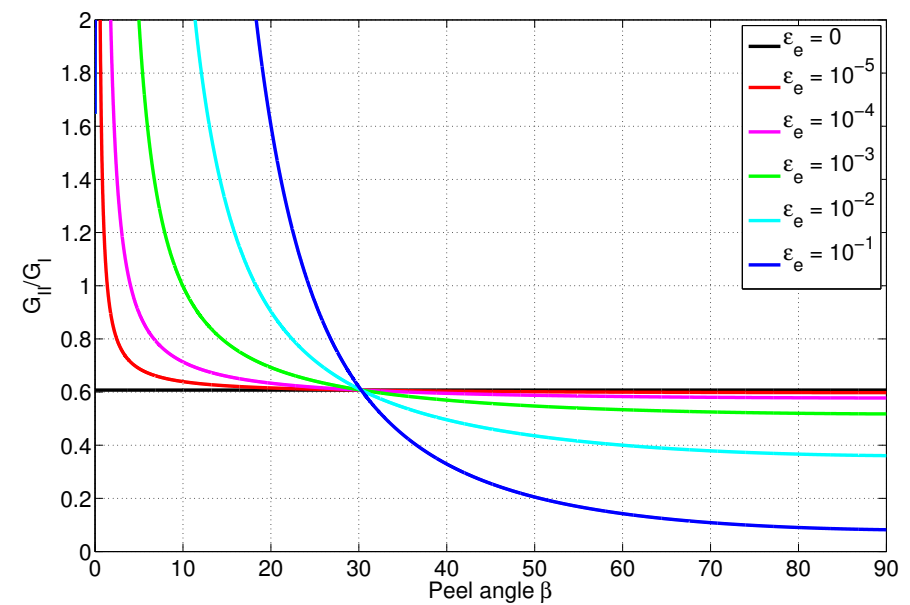

Figure 5: Mode ratio as a function of the peel angle for different values of the dimensionless peel force (elastic tensile strain) in the peel arm 


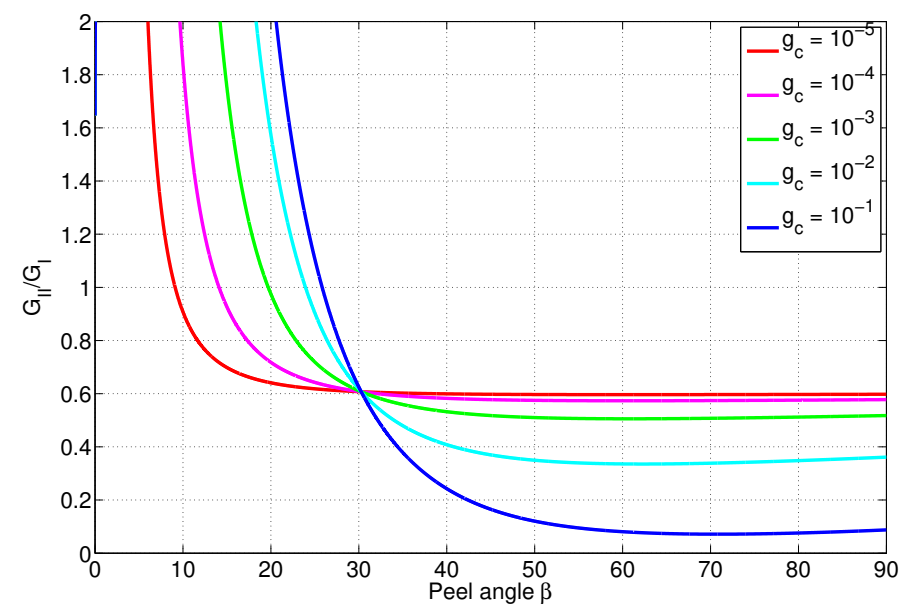

Figure 6: Mode ratio as a function of the peel angle for different values of the dimensionless critical strain energy release rate

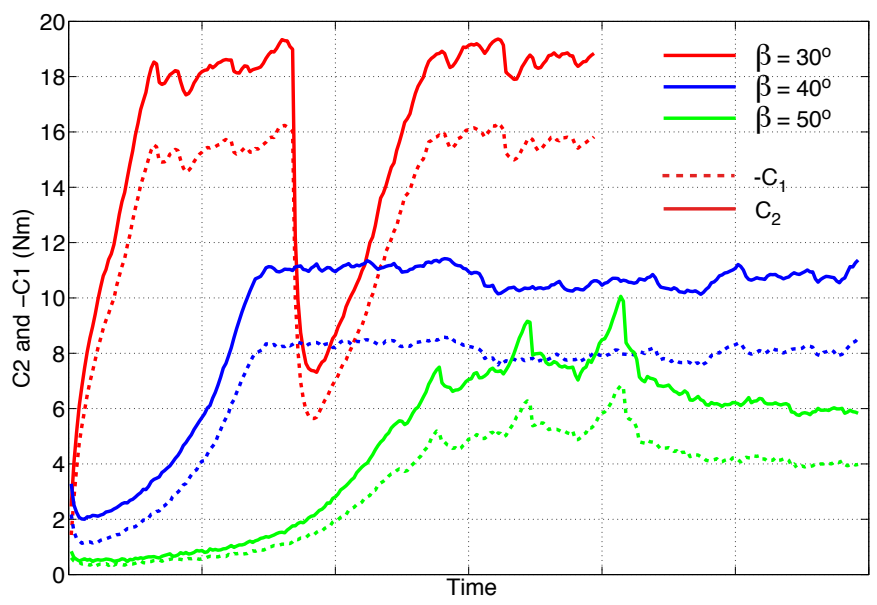

Figure 7: Measures of the couples $-C_{1}$ and $C_{2}$ for different peel angles

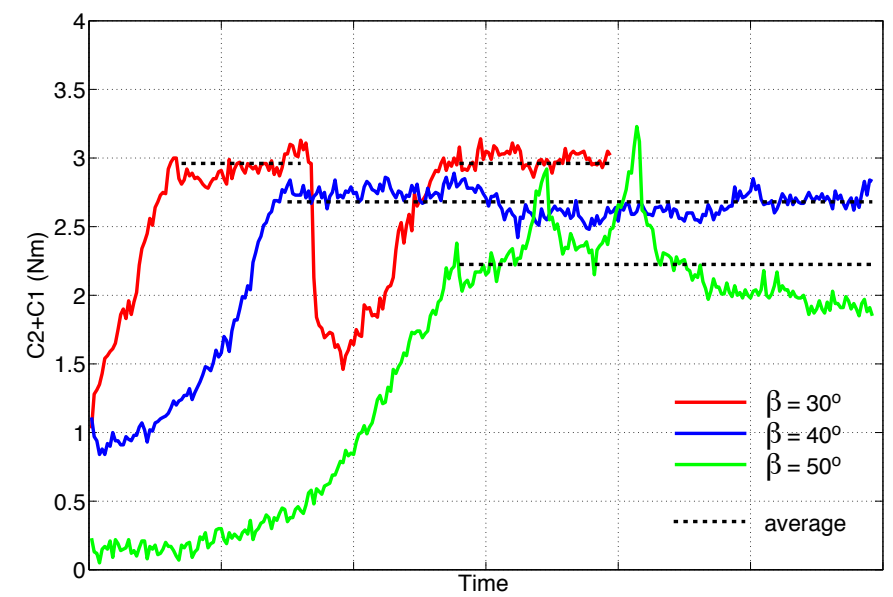

Figure 8: Plot of $C_{1}+C_{2}$ for different peel angles and average values used for the computation of $G_{c}$ 


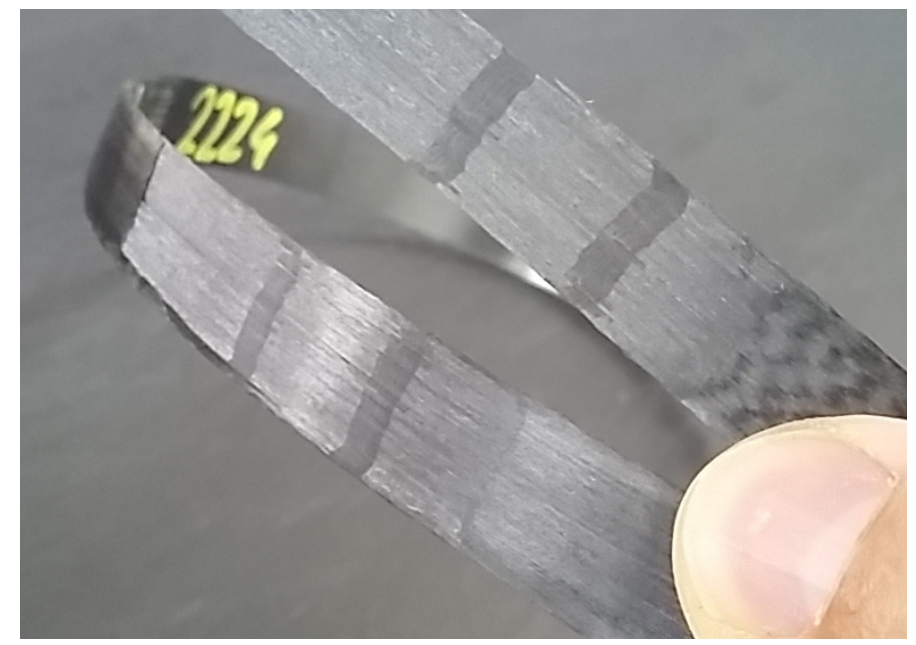

Figure 9: Fracture surface for a test with $\beta=30^{\circ}$ : progressive (light) and unstable (dark) portions of the delamination

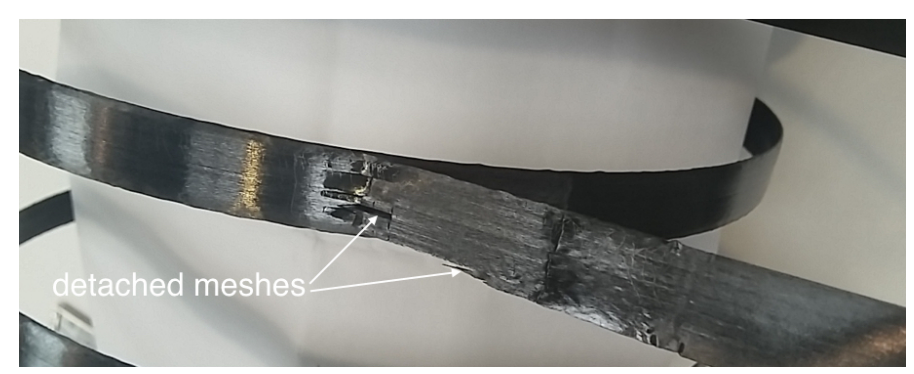

Figure 10: Damage in the peel arm after testing at $\beta=50^{\circ}$

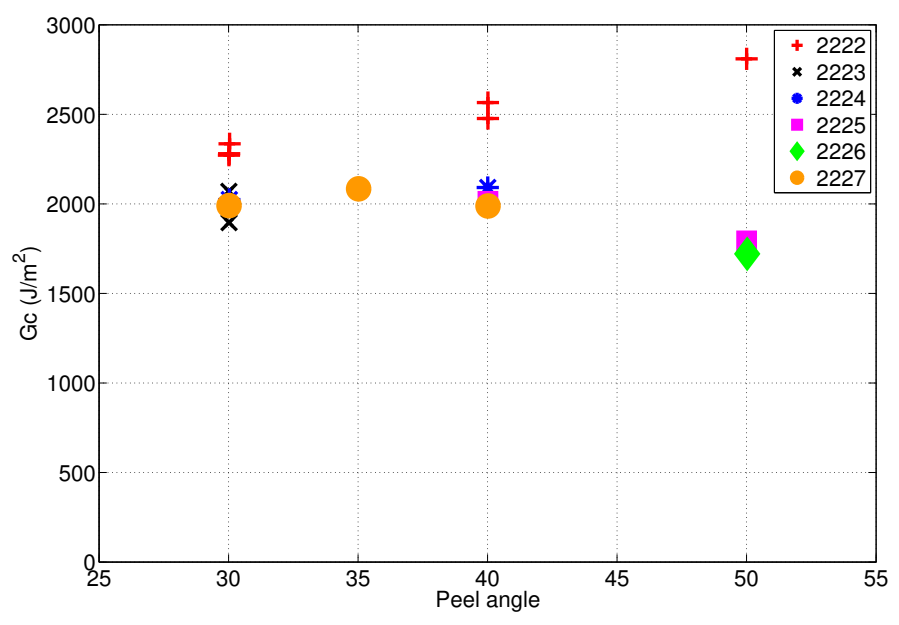

Figure 11: $G_{c}$ as a function of the peel angle for all the DDP tests 